\title{
Exact pp-waves, (A)dS waves, and Kundt spaces in the Abelian-Higgs model
}

\author{
Fabrizio Canfora, ${ }^{1, *}$ Adolfo Cisterna, ${ }^{2, \dagger}$ Diego Hidalgo, ${ }^{1,3,4, \$}$ and Julio Oliva ${ }^{3, \S}$ \\ ${ }^{1}$ Centro de Estudios Científicos (CECs), Casilla 1469, Valdivia, Chile \\ ${ }^{2}$ Sede Esmeralda, Universidad de Tarapacá, Av. Luis Emilio Recabarren 2477, Iquique, Chile \\ ${ }^{3}$ Departamento de Física, Universidad de Concepción, Casilla 160-C, Concepción, Chile \\ ${ }^{4}$ Instituto de Ciencias Físicas y Matemáticas, Universidad Austral de Chile, Casilla 567, Valdivia, Chile
}

(Received 15 March 2021; accepted 18 March 2021; published 20 April 2021)

\begin{abstract}
We find new exact solutions of the Abelian-Higgs model coupled to general relativity, characterized by a nonvanishing superconducting current. The solutions correspond to $p p$-waves, AdS waves, and Kundt spaces, for which both the Maxwell field and the gradient of the phase of the scalar are aligned with the null direction defining these spaces. In the Kundt family, the geometry of the two-dimensional surfaces orthogonal to the superconducting current is determined by the solutions of the two-dimensional Liouville equation, and in consequence, these surfaces are of constant curvature, as it occurs in a vacuum. The solution to the Liouville equation also acts as a potential for the Maxwell field, which we integrate into a closed-form. Using these results, we show that the combined effects of the gravitational and scalar interactions can confine the electromagnetic field within a bounded region in the surfaces transverse to the current.
\end{abstract}

DOI: 10.1103/PhysRevD.103.085007

\section{INTRODUCTION}

A very important step toward a deep understanding of a classical field theory is a proper understanding of its classical solutions. For a generic field theory, this may seem an insurmountable task since the space of solutions are infinite-dimensional, nevertheless, for general relativity (GR), important classification schemes are available which allow defining classes of solutions, contributing to the understanding of their potential realization in nature [1].

One of the most relevant field theory (both at the classical and quantum level) is the Abelian-Higgs model (the Maxwell-Ginzburg-Landau theory) which can describe successfully many important semiclassical features of superconductors (see [2,3] for detailed reviews: in the following, we will consider the relativistic version of the theory). A further important phenomenological implication of this theory is the presence of vortices discovered by Abrikosov, Nielsen and Olesen in [4,5]. These are some of the many reasons why the minimal coupling of the AbelianHiggs model with GR has been deeply investigated (see [3] and references therein). Moreover, a no-hair theorem was

\footnotetext{
*anfora@cecs.cl

†adolfo.cisterna@ucentral.cl

¥dhidalgo@ cecs.cl

§uoliva@udec.cl
}

Published by the American Physical Society under the terms of the Creative Commons Attribution 4.0 International license. Further distribution of this work must maintain attribution to the author(s) and the published article's title, journal citation, and DOI. Funded by SCOAP ${ }^{3}$. proved in [6], which can be circumvented for horizons pierced by a vortex both in the static case [7], as well as for stationary black holes [8], and for planar AdS black holes [9]. In the holographic setup, this system allows constructing holographic superconductors, where near the horizon of a black hole the scalar acquires a vev [10-13], which can be understood as arising due to an instability triggered by a violation of the effective Breitenlohner-Freedman bound [14] in the $\mathrm{AdS}_{2}$ near horizon geometry of extremal Reissner-Nordström black hole. Finally, this system also finds applications in the holographic description of superfluidity (see, e.g., $[13,15,16])$. Given the relevance of this field theory, it is of uttermost importance to continue shedding light on the structure of its space of solutions. This paper is devoted to such a task. In the present manuscript, we will study, with analytic methods, the gravitational consequences of the presence of a superconducting current in the Einstein-Maxwell-GinzburgLandau theory.

Of course, one may wonder why to insist on finding analytic solutions if these equations can be solved numerically. Indeed, numerical techniques were already available in the literature of the eighties and nineties to analyze these configurations in the gravitating Abelian-Higgs model (see [3] and references therein). Despite this, there are indisputable arguments that strongly suggest that, whenever it is possible, we should strive for analytic solutions. For example, much of what we currently know about black hole physics in GR, and instantons and monopoles in gauge theories arose from a careful study of the available analytic solutions like the Kerr solution in the former and non-Abelian monopoles and instantons in the latter. 
Consequently, an analytic tool to analyze the gravitational effects of superconducting currents in the model relevant to our present study can greatly enlarge our understanding of this system. Second and more concretely, our analysis discloses a nice mechanism that, at least in principle, can confine the electromagnetic field in the two-dimensional surfaces orthogonal to the superconducting currents.

One may think that pursuing an analytic approach in this nonlinear system is hopeless. Nevertheless, the methods developed in [17-22] to propose a proper ansatz, allowed to construct analytic gauged solitons in the gauged Skyrme model thanks to a suitable choice of variables which enables to partially decouple the field equations. These were extended in [23] to include the minimal coupling with GR and here we show that they are suitable to analyze Einstein-Maxwell-Ginzburg-Landau theory, as well.

In Sec. II, we present the model and describe properties of the superconducting current supporting the solutions of the following sections. In Sec. III, we construct the $p p$ wave as well as the AdS-wave solutions and for the latter, in a particular case, we can integrate the whole system in an explicit, closed manner. Section IV is devoted to the construction of the Kundt solutions, characterized by the existence of a null, geodesic, congruence that is not covariantly constant but has vanishing optical scalars. The Liouville equation naturally emerges in the constant $u, v$ sector and we obtain nontrivial solutions for both, the positive and negative cosmological constant value. Finally, we provide some conclusions in Sec. V.

\section{THE MODEL}

The gravitating Abelian-Higgs model is described by the action

$$
\begin{aligned}
S[g, \Psi, A]= & \int d^{4} x \sqrt{-g}\left(R-2 \Lambda-\frac{1}{4} F_{\mu \nu} F^{\mu \nu}\right. \\
& \left.-D_{\mu} \Psi\left(D^{\mu} \Psi\right)^{\star}-V(\Psi)\right),
\end{aligned}
$$

where $g$ is the determinant of the metric, $R$ is the Ricci tensor scalar, $\Lambda$ the cosmological constant, and we have set $16 \pi G=1$. The scalar field $\Psi$ is complex, and $\Psi^{\star}$ denotes its complex conjugate. The electromagnetic field strength is given by $F_{\mu \nu}=\partial_{\mu} A_{\nu}-\partial_{\nu} A_{\mu}$, with $A_{\mu}$ the electromagnetic potential. In (1), we have introduced the gauge covariant derivative of the field with charge $q$ and its conjugate with charge $-q$ as

$$
\begin{aligned}
D_{\mu} \Psi & =\partial_{\mu} \Psi+i q A_{\mu} \Psi, \\
\left(D_{\mu} \Psi\right)^{\star} & =\partial_{\mu} \Psi^{\star}-i q A_{\mu} \Psi^{\star},
\end{aligned}
$$

and hereafter $\nabla_{\mu}$ denotes the covariant derivative constructed with the Christoffel symbol. In the Abelian-Higgs model, the self-interacting potential $V(\Psi)$ of the complex scalar field is given by

$$
V(|\Psi|)=\lambda\left(\Psi \Psi^{\star}-\nu_{0}^{2}\right)^{2}
$$

where $\nu_{0}$ is a real constant and $\lambda>0$. The field equations that follows from varying the action (1) are

$$
\begin{gathered}
R_{\mu \nu}-\frac{1}{2} g_{\mu \nu} R+\Lambda g_{\mu \nu}=T_{\mu \nu}, \\
\nabla_{\mu} F^{\mu \nu}=J^{\nu}, \\
\nabla_{\mu} \nabla^{\mu} \Psi+i q \nabla_{\mu} A^{\mu} \Psi+2 i q A^{\mu} \nabla_{\mu} \Psi \\
-q^{2} A_{\mu} A^{\mu} \Psi-\frac{\partial}{\partial \Psi^{\star}} V(|\Psi|)=0 .
\end{gathered}
$$

The stress-energy tensor $T_{\mu \nu}$ is the sum of two contributions

$$
T_{\mu \nu}=T_{\mu \nu}^{(A)}+T_{\mu \nu}^{(\Psi)}
$$

associated to the Maxwell and the scalar field, respectively, given by

$$
\begin{aligned}
T_{\mu \nu}^{(A)} & =\frac{1}{2}\left(F_{\mu \alpha} F_{\nu}{ }^{\alpha}-\frac{1}{4} g_{\mu \nu} F_{\alpha \beta} F^{\alpha \beta}\right), \\
T_{\mu \nu}^{(\Psi)}= & \frac{1}{2}\left(D_{\mu} \Psi\left(D_{\nu} \Psi\right)^{\star}+D_{\nu} \Psi\left(D_{\mu} \Psi\right)^{\star}\right. \\
& \left.-g_{\mu \nu}\left(D_{\alpha} \Psi\left(D^{\alpha} \Psi\right)^{\star}+V(\Psi)\right)\right) .
\end{aligned}
$$

In (4b), the particle number current is given by

$$
J_{\mu}=i q\left(\left(D_{\mu} \Psi\right)^{\star} \Psi-D_{\mu} \Psi \Psi^{\star}\right) .
$$

In the following, we focus on two new families of independent solutions to this model. First, we construct new charged $p p$-waves and (A)dS waves and show that they are controlled by an integrable system. Then, inspired by an extension of these solutions we will construct new charged spacetimes that contain a two-dimensional sector whose conformal factor leads to the Liouville equation in two dimensions. We will see that this function plays the role of a potential and source of the Maxwell equation and the remaining Einstein equations, respectively.

Before proceeding with the construction of the exact solutions, a few remarks are in order regarding the persistent character of the $U(1)$ currents. In [24] the deep and consequential idea of superconducting strings was proposed. This idea (which was further generalized, for instance, in [25-48] and references therein) shed light on the highly nontrivial gravitational effects of superconducting currents. These references partly motivated the present analysis to build the simplest possible analytic example of gravitational fields sourced by currents with the characteristics listed here below. As far as the present analysis is 
concerned, the relevant features of the $U(1)$ persistent current [24] for our construction are

(i) The $U(1)$ current (whose gravitational effects are under examination) should survive even in the limit of zero gauge potential.

(ii) The corresponding residual current $J_{(0) \mu}$ (in the limit $A_{\mu}=0$ ) should have the form

$$
J_{(0) \mu}=\Gamma \partial_{\mu} \Omega,
$$

where $\Gamma$ is a function which cannot vanish everywhere while the function $\Omega$ is defined only modulo $2 \pi: \Omega \sim \Omega+2 \pi$.

As far as the function $\Gamma$ is concerned, the simplest case corresponds to $\Gamma=$ cte: in the following, we will consider configurations in which this option is realized. While for the function $\Omega$, we will consider configurations in which the fact that $\Omega$ is defined only modulo $2 \pi$ is manifest. In particular, from Eqs. (11) and (27) below it is clear that the function $\Omega$ is defined only modulo $2 \pi$ and that the current is proportional to $\partial_{\mu} \Omega$.

\section{PP AND AdS WAVES}

In order to simplify the presentation of the new solutions obtained in this section, we separate the analysis of the $\Lambda=0$ case, from that with nonvanishing $\Lambda$.

\section{A. $p p$-waves}

The metric for a $p p$-wave in Brinkmann coordinates reads

$$
d s^{2}=-F(u, x, y) d u^{2}-2 d u d v+d x^{2}+d y^{2} .
$$

This geometry is characterized by possessing a covariantly constant vector $\partial_{v}$, which being nontwisting, is orthogonal to the two-dimensional, planar hypersurface spanned by the coordinates $(x, y)$. In vacuum, Einstein equations imply that the wave profile $F(u, x, y)$ can be separated as an arbitrary function of the coordinate $u$, times a harmonic function on $(x, y)$. On the other hand, these spacetimes are consistent with the backreaction produced for example by a conformal source [49]. Metrics of the form (10) also play an important role in holography since they emerge, for example, as supersymmetric configurations by taking a suitable Penrose limit of the $\mathrm{AdS}_{5} \times S^{5}$ solution of Type-IIB SUGRA [50].

Here, in the context of the gravitating Abelian-Higgs model (1), we focus on the spontaneously broken phase, but maintaining the phase of the scalar turned on, and we impose that both, the gradient of the scalar $\partial_{\mu} \psi$ as well as the gauge field $A_{\mu}$, to be aligned with the covariantly constant vector $\partial_{v}$ that defines the $p p$-wave (10). This kind of strategy to decouple the field equations describing gauged solitons in the low energy limit of QCD, minimally coupled to Maxwell equations, has been introduced in [17-23]. These conditions lead to

$$
\begin{gathered}
\psi=\rho e^{i \Omega(u)}, \quad \text { and } \quad A=a(u, x, y) d u, \\
A^{2}=\nabla_{\mu} A^{\mu}=A \cdot \nabla \psi=0,
\end{gathered}
$$

which have also been useful in the construction of static and rotating solutions in vector Galileon theories [51].

Under these circumstances, considering a constant value of $\rho=\nu_{0}=c t e$, we obtain an effective system of equations given by

$$
\begin{aligned}
G_{\mu \nu}= & \frac{1}{2} F_{\mu \lambda} F_{\nu}^{\lambda}-\frac{1}{8} g_{\mu \nu} F^{2} \\
& +\rho^{2}\left[\nabla_{\mu} \Omega \nabla_{\nu} \Omega+q\left(A_{\mu} \nabla_{\nu} \Omega+A_{\nu} \nabla_{\mu} \Omega\right)+q^{2} A_{\mu} A_{\nu}\right] \\
& \nabla_{\mu} F^{\mu \nu}=2 q \rho^{2} \nabla^{\nu} \Omega+2 q^{2} \rho^{2} A^{\nu}
\end{aligned}
$$

and the Klein-Gordon equation is automatically satisfied when $\rho$ is constant. As expected, on the spontaneously broken phase, the vector field $A_{\mu}$ acquires a mass which can be read from (14) leading to

$$
m_{\mathbf{A}}^{2}=2 q^{2} \rho^{2} .
$$

Defining $\omega(u)=\partial_{u} \Omega(u)$, one can show that the whole system for the gravitating Abelian-Higgs model, in this sector reduces to the following two equations

$$
\left(\frac{\partial^{2}}{\partial x^{2}}+\frac{\partial^{2}}{\partial y^{2}}\right) a(u, x, y)-2 q \rho^{2}(q a(u, x, y)+\omega(u))=0,
$$

$$
\begin{aligned}
& \left(\frac{\partial^{2}}{\partial x^{2}}+\frac{\partial^{2}}{\partial y^{2}}\right) F(u, x, y)-\left(\frac{\partial}{\partial x} a(u, x, y)\right)^{2} \\
& -\left(\frac{\partial}{\partial y} a(u, x, y)\right)^{2}-2 \rho^{2}(q a(u, x, y)+\omega(u))^{2}=0 .
\end{aligned}
$$

Remarkably, we have arrived to an integrable system. The equation (16) is a screened Poisson equation for the gauge field component $a(u, x, y)$, which can be integrated in terms of a convolution of the Green function for this operator and the source $\omega(u)$, which is the phase of the complex scalar field. Clearly, the effective mass of the vector field $m_{\mathbf{A}}$ given in (15) is responsible for the screening. Once this equation is integrated, Eq. (17) transforms into a Poisson equation for the $p p$-wave profile $F(u, x, y)$, which again, can be integrated using the corresponding Green function.

Notice that one may want to remove the phase $\omega(u)$ by a gauge transformation $\psi \rightarrow \psi e^{-i q \xi\left(x^{\mu}\right)}$ and $A_{\mu} \rightarrow A_{\mu}+\partial_{\mu} \xi$. 
This can be achieved locally, but since the phase depends on the null direction $u$, in order to remove it, one must in general implement a large gauge transformation. Such transformations can modify the global interpretation of the solution, thus we prefer not to remove $\omega(u)$.

\section{B. (A)dS waves}

In this case the spacetime takes the form

$d s^{2}=\frac{\ell^{2}}{x^{2}}\left(-F(u, x, y) d u^{2}-2 d u d v+d x^{2}+d y^{2}\right)$,

where we have defined $\Lambda=-3 / \ell^{2}$, with $\ell$ the AdS radius. These Siklos spacetimes correspond to a conformal transformation of the $p p$-wave (10). Now, the field equations reduce to:

$$
\begin{gathered}
x^{4}\left(\frac{\partial^{2}}{\partial x^{2}}+\frac{\partial^{2}}{\partial y^{2}}\right) a(u, x, y) \\
-2 x^{2} \ell^{2} q \rho^{2}(q a(u, x, y)+\omega(u))=0, \\
\left(\frac{\partial^{2}}{\partial x^{2}}+\frac{\partial^{2}}{\partial y^{2}}-\frac{2}{x} \frac{\partial}{\partial x}\right) F(u, x, y) \\
-\frac{x^{2}}{\ell^{2}}\left(\left(\frac{\partial}{\partial x} a(u, x, y)\right)^{2}+\left(\frac{\partial}{\partial y} a(u, x, y)\right)^{2}\right) \\
-2 \rho^{2}(q a(u, x, y)+\omega(u))^{2}=0 .
\end{gathered}
$$

In the presence of the cosmological term, the Maxwell equation (19) is not an autonomous equation anymore, nevertheless it can be integrated and leads to

$$
\begin{aligned}
a(u, x, y)= & \omega(u)\left[x ^ { 1 / 2 } \left(A(y) x^{\nu}+B(y) x^{-\nu}\right.\right. \\
& \left.\left.+\left(C_{1} J_{\nu}(c x)+C_{2} Y_{\nu}(c x)\right)\left(C_{3} e^{c y}+C_{4} e^{-c y}\right)\right)-\frac{1}{q}\right],
\end{aligned}
$$

where $J_{\nu}$ and $Y_{\nu}$ are the Bessel functions of the first and second kind, respectively, $A(y)$ and $B(y)$ are arbitrary linear functions of $y$ and $C_{i=1, \ldots, 4}$ and $c$ are integration constants. We have also defined

$$
\nu=\frac{1}{2} \sqrt{1+8 \ell^{2} q^{2} \rho^{2}} .
$$

Even though we have been able to integrate the Maxwell's equation in a closed form, for nonvanishing constants $C_{i}$, the metric profile $F(u, x, y)$ cannot be integrated in a closed manner. To move forward, we therefore set $C_{1}=C_{2}=0$ as well as $A(y)=A_{0}$ and $B(y)=B_{0}$. Under these conditions the electromagnetic field (21) reduces to

$$
a(u, x, y)=\omega(u)\left[x^{1 / 2}\left(A_{0} x^{\nu}+B_{0} x^{-\nu}\right)-\frac{1}{q}\right],
$$

and the AdS-wave profile reads,

$$
\begin{aligned}
F(u, x, y)= & \omega(u)^{2}\left[\left(D_{1} e^{h x}(1-h x)+D_{2} e^{-h x}(1+h x)\right)\left(D_{4} \sin (h y)+D_{5} \cos (h y)\right)\right. \\
& \left.+\frac{1}{8}\left(E_{1}+E_{2} x^{3}+\frac{1}{\ell^{2}}\left(A_{0}^{2} \frac{(1+2 \nu)}{(3+2 \nu)} x^{3+2 \nu}+B_{0}^{2} \frac{(2 \nu-1)}{(2 \nu-3)} x^{3-2 \nu}\right)\right)\right] .
\end{aligned}
$$

Here again, $D_{1, \ldots, 5}, h$ and $E_{1,2}$ are integration constants. We can see that the effect of the charge on the function $F(u, x, y)$ induces a quite nontrivial profile.

Before finishing this section, it is interesting to notice that the expression for $\nu$ in (22) can be written in terms of the effective mass of the vector field on the broken phase, $m_{\mathbf{A}}$ given in (15), as

$$
2 \nu=\sqrt{1-\frac{m_{\mathbf{A}}^{2}}{m_{\mathbf{B F}}^{2}}},
$$

where $m_{\mathbf{B F}}^{2}=-(2 \ell)^{-2}$ is the Breitenlohner-Freedman bound for a spin 1 field on $\mathrm{AdS}_{4}$. Therefore, as expected, the $x$ dependence of Eq. (23) is reminiscent of that for a massive vector on AdS (see, e.g., equation (25)-(26) of [52]).

\section{KUNDT SPACES IN THE ABELIAN-HIGGS MODEL}

Let us consider now consider an extension of the $p p$-wave ansatz, given by

$$
\begin{aligned}
d s^{2}= & \left(f(x, y)+f_{0} v+\Lambda v^{2}\right) d u^{2}-2 d v d u \\
& +e^{\beta h(x, y)}\left(d x^{2}+d y^{2}\right)
\end{aligned}
$$

where $u=t+w, v=t-w$ is a null coordinate, $x, y, w$ are Cartesian-like coordinates, and $f_{0}$ and $\beta$ are arbitrary constants. This spacetime belongs to the Kundt family since it can be checked that the null congruence generated by $\partial_{v}$ is not covariantly constant, but nevertheless it has vanishing expansion, shear, and twist.

The techniques developed in [17-22] are particularly suitable to analyze gravitating solitons whose metrics have 
the form in Eq. (26) (see the analysis in [23]). Again, the complex scalar field adopts a harmonic dependence in $u$, and with a constant amplitude, given by

$$
\Psi\left(x^{\mu}\right)=\nu_{0} e^{i \Omega(u)}, \quad \Omega(u)=u .
$$

We also assume that the Maxwell field has the following form

$$
A=A_{u}(x, y) d u, \quad A_{u}(x, y)=\frac{a(x, y)-1}{q} .
$$

With this ansatz, the Klein-Gordon (4c) is automatically satisfied, while Maxwell equations reduce to

$$
\left(\frac{\partial^{2}}{\partial x^{2}}+\frac{\partial^{2}}{\partial y^{2}}-2 q^{2} \nu_{0}^{2} e^{\beta h(x, y)}\right) a(x, y)=0 .
$$

The only nontrivial Einstein field equations (4a) for this configuration are

$$
\begin{gathered}
\left(\frac{\partial^{2}}{\partial x^{2}}+\frac{\partial^{2}}{\partial y^{2}}\right) h(x, y)=-\frac{2 \Lambda}{\beta} e^{\beta h(x, y)} \\
\left(\frac{\partial^{2}}{\partial x^{2}}+\frac{\partial^{2}}{\partial y^{2}}\right) f(x, y)=-\rho(x, y)
\end{gathered}
$$

with

$$
\begin{aligned}
\rho(x, y)= & 2 \nu_{0}^{2} e^{\beta h(x, y)} a^{2}(x, y) \\
& +\frac{1}{q^{2}}\left(\left(\frac{\partial a(x, y)}{\partial x}\right)^{2}+\left(\frac{\partial a(x, y)}{\partial y}\right)^{2}\right) .
\end{aligned}
$$

Equation (30) correspond to a Liouville equation for $h(x, y)$, namely the conformal factor of the two-dimensional space spanned by the coordinates $(x, y)$ in the metric (26), and a Poisson equation for the function $f(x, y)$. Thus, as it happens in [23] in the case of Einstein-Maxwell coupled to a nonlinear sigma model, the present ansatz allows a useful partial decoupling of the field equations. In particular, Eq. (30a) allows a direct integration for $h(x, y)$. This equation actually implies that the induced metric on the $u, v=$ constant surfaces is of constant curvature $\Lambda$, as it occurs in vacuum [1]. Then, once $h(x, y)$ is known, one can solve the Maxwell equation in Eq. (29) for $a(x, y)$ since it reduces to a Schrödinger-like equation in which $e^{\beta h(x, y)}$ plays the role of the potential. Eventually, once $h(x, y)$ and $a(x, y)$ are both known, one can solve the remaining equation, Eq. (30b) for $f(x, y)$, since the source term $\rho(x, y)$ is explicitly known once $h(x, y)$ and $a(x, y)$ determined. This hierarchical decoupling is the key of the strategy developed in [17-22]. Therefore, following this logic, we start considering the general solution of $(30 a)$, given by $[53,54]$

$$
\begin{aligned}
& e^{\beta h(x, y)}=\frac{4}{\Lambda} \frac{g^{\prime}(z) \bar{g}^{\prime}(\bar{z})}{(g(z) \bar{g}(\bar{z})+1)^{2}}, \quad \text { if } \Lambda>0, \\
& e^{\beta h(x, y)}=-\frac{4}{\Lambda} \frac{g^{\prime}(z) \bar{g}^{\prime}(\bar{z})}{(g(z) \bar{g}(\bar{z})-1)^{2}}, \quad \text { if } \Lambda<0,
\end{aligned}
$$

where $g(z)$ is any meromorphic function of $z=x+i y$, with at most simple poles, and $d g / d z \neq 0$ for all $z$ in a simply connected domain. On the other hand, since the coordinates $x, y$ are Cartesian, the Poisson equation possesses the particular solution [55]

$$
\begin{aligned}
f(x, y)= & \frac{1}{2 \pi} \int_{-\infty}^{\infty} \int_{-\infty}^{\infty} \rho(\bar{x}, \bar{y}) \ln \left(\frac{1}{\sqrt{(x-\bar{x})^{2}+(y-\bar{y})^{2}}}\right) \\
& \times d \bar{x} d \bar{y} .
\end{aligned}
$$

As mentioned, the Liouville equation on $h(x, y)$, implies that the manifold spanned by the coordinates $(x, y)$ is of constant curvature $\Lambda$. Therefore, locally, there is always a change of coordinates that allows rewriting the metric (26) as

$$
\begin{aligned}
d s^{2}= & \left(f(\mu, \phi)+f_{0} v+\Lambda v^{2}\right) d u^{2}-2 d v d u \\
& +\frac{d \mu^{2}}{1-\Lambda \mu^{2}}+\mu^{2} d \phi^{2} .
\end{aligned}
$$

In these coordinates, the equation for the electromagnetic field $a(r, \theta)$ reads

$$
\begin{aligned}
& \frac{d^{2} a(\mu, \phi)}{d \mu^{2}}+\frac{\left(1-2 \Lambda \mu^{2}\right)}{\mu\left(1-\Lambda \mu^{2}\right)} \frac{d a(\mu, \phi)}{d \mu}-\frac{2 q^{2} \nu_{0}^{2} a(\mu, \phi)}{\left(1-\Lambda \mu^{2}\right)} \\
& +\frac{1}{\mu^{2}\left(1-\Lambda \mu^{2}\right)} \frac{d^{2} a(\mu, \phi)}{d \phi^{2}}=0 .
\end{aligned}
$$

The general solution to this equation is

$$
a(\mu, \phi)=\sum_{m} a_{m} \sin \left(m \phi+\delta_{m}\right) G_{m}(\mu),
$$

where the function $G_{m}(\mu)$ can be integrated in terms of Legendre functions. Here $a_{m}$ and $\delta_{m}$ are integration constants.

For the $\Lambda<0$ case, setting $\Lambda=-1$, the radial coordinate $\mu$ goes from $\left[0, \infty\left[\right.\right.$, and the solution for $G_{m}(\mu)$ which is nondivergent as $\mu \rightarrow \infty$ reads

$$
\begin{aligned}
G_{m}^{\Lambda<0}(\mu) & \\
& =\mu^{-\frac{1}{2}(1+2 \nu)}{ }_{2} F_{1}\left(-\frac{m}{2}+\frac{\nu}{2}+\frac{1}{4}, \frac{m}{2}+\frac{\nu}{2}+\frac{1}{4}, 1+\nu,-\mu^{-2}\right),
\end{aligned}
$$

where ${ }_{2} F_{1}$ stands for the Gauss hypergeometric function and $\nu$ was defined in (22). In this case the two-dimensional surfaces at $u, v=$ constant are hyperbolic spaces with 
origin at $\mu=0$. Even though the behavior at $\mu \rightarrow \infty$ is regular, these solutions have a singular behavior near the center $\mu=0$ of the hyperbolic space, since near such point, one can see that

$$
G_{m}^{\Lambda<0}(\mu)=A \mu^{m}(1+\mathcal{O}(\mu))+B \mu^{-m}(1+\mathcal{O}(\mu)),
$$

and one can see that both constant $A, B$ are always nonvanishing. In spite of this behavior, one can check that the curvature invariants $R, R_{\alpha \beta \gamma \delta} R^{\alpha \beta \gamma \delta}, R_{\alpha \beta}{ }^{\gamma \delta} R_{\gamma \delta}{ }^{\tau \sigma} R_{\tau \sigma}{ }^{\alpha \beta}$ are actually constant, therefore there is no singular backreaction on the geometry. Interestingly, the equation for $G_{m}$ in this case can be written as a Schrödinger-like equation of the form

$$
-\frac{d^{2} G_{m}(s)}{d s^{2}}+\frac{2 q^{2} \nu_{0}^{2}}{\sinh ^{2}(s)} G_{m}(s)=-m^{2} G_{m}(s),
$$

where we have introduced the inversion $\mu=(\sinh (s))^{-1}$ which maps the range $\mu \in(0, \infty)$ to $s \in(\infty, 0)$. This is a Schröedinger-like equation in a generalized Pöschl-Teller potential, which belongs to a class of exactly solvable, shape invariant potentials [56,57]. The potential being positive, clearly implies that there cannot be solutions that are regular at both boundaries of the domain $s \in(\infty, 0)$, which is consistent with the asymptotic expansion of (37) around $\mu=0$ presented in (38). Nevertheless, as also mentioned above, the backreaction on the geometry of this Maxwell field is regular.

When $\Lambda>0$, the range of the $\mu$-coordinate in (34) is $\mu \in]-1,1[$. Setting $\Lambda=1$ in this case, and defining $\mu=\sin (\theta)$, leads to the following solution

$$
\begin{aligned}
G_{m}^{\Lambda>0}= & \sin ^{|m|}(\theta)_{2} F_{1}\left(\frac{|m|}{2}-\frac{1}{4} \sqrt{1-8 q^{2} \nu_{0}^{2}}+\frac{1}{4},\right. \\
& \left.\frac{|m|}{2}+\frac{1}{4} \sqrt{1-8 q^{2} \nu_{0}^{2}}+\frac{1}{4}, 1+|m|, \sin ^{2}(\theta)\right),
\end{aligned}
$$

which is regular at the poles located at $\theta=0$ and $\theta=\pi$.

Finally, it is also instructive to see explicitly how these cases emerge from a suitable choice of the arbitrary function $g(z)$ of the general solution of the Liouville equation in (32) and (32a). For concreteness, let us focus on the case with negative cosmological constant, normalized as $\Lambda=-1$, namely the case corresponding to Eq. (32a). Choosing $g(z)=z$ and $\bar{g}(\bar{z})=\bar{z}$ in (32a) leads to the following metric for the Kundt spaces

$$
\begin{aligned}
d s^{2}= & \left(f(x, y)+f_{0} v+\Lambda v^{2}\right) d u^{2}-2 d v d u \\
& +\frac{4\left(d x^{2}+d y^{2}\right)}{\left(1-\left(x^{2}+y^{2}\right)\right)^{2}}
\end{aligned}
$$

which after the change of coordinates

$$
\begin{aligned}
& x=\mu^{-1}\left(\sqrt{1+\mu^{2}}-1\right) \cos (\phi), \\
& y=\mu^{-1}\left(\sqrt{1+\mu^{2}}-1\right) \sin (\phi),
\end{aligned}
$$

leads to the metric

$$
\begin{aligned}
d s^{2}= & \left(f(x, y)+f_{0} v+\Lambda v^{2}\right) d u^{2}-2 d v d u \\
& +\frac{d \mu^{2}}{1+\mu^{2}}+\mu^{2} d \phi^{2}
\end{aligned}
$$

that we have used in (34) and (35).

\section{CONCLUSIONS}

We have constructed three new families of analytic solutions of the gravitating Abelian-Higgs model, characterized by a nonvanishing superconducting current. The first two families of solutions correspond to exact gravitational waves: $p p$ and (A)dS waves. In these families the null vector characterizing both the $p p$-wave and the (A) $\mathrm{dS}$-wave is aligned with the superconducting current. Then, we have studied a class of solutions that belong to the family of Kundt spaces, and as in vacuum, the twodimensional geometry of the surfaces orthogonal to the superconducting currents is determined by the twodimensional Liouville equation. Such surfaces can have either positive or negative Gaussian curvature depending on the sign of the cosmological constant. ${ }^{1}$ This sector possesses a remarkable property: the arbitrary analytic function characterizing the solution of the two-dimensional Liouville equation (which determines the geometry of twodimensional surfaces transverse to the superconducting current) can be chosen in such a way that the corresponding Maxwell equations reduce consistently to a Schrödingerlike equation in a generalized Pöschl-Teller potential. Requiring suitable boundary conditions for the Maxwell field within this sector, for a negative cosmological constant, the combined effects of the gravitational and scalar interactions can confine the electromagnetic field within a bounded region of the surfaces transverse to the current itself. This result opens the interesting possibility to analyze the properties of test electromagnetic fields propagating within these families of analytic solutions of the AbelianHiggs model using the well known properties of the PöschlTeller potential [57]. We hope to come back on this feature in the future.

\section{ACKNOWLEDGMENTS}

We thank Eloy Ayón-Beato and Francisco Correa for enlightening comments on related topics. F. C., A. C., and

\footnotetext{
${ }^{1}$ It would be nice constructing an exhaustive classification of Kundt solutions in this model, along the lines of [58].
} 
J. O. have been funded by Fondecyt Grants No. 1200022, No. 1210500 , and No. 1181047 , respectively. D. H. is partially founded by ANID Grant No. 21160649. The Centro de Estudios Científicos (CECs) is funded by the
Chilean Government through the Centers of Excellence Base Financing Program of ANID. This work is also partially funded by Proyecto de Cooperación Internacional 2019/13231-7 FAPESP/ANID.
[1] H. Stephani, D. Kramer, M. A. H. MacCallum, C. Hoenselaers, and E. Herlt, Exact solutions of Einstein's field equations; J. B. Griffiths and J. Podolsky, Exact Space-Times in Einstein's General Relativity (Cambridge University Press, Cambridge, England, 2009).

[2] N. Manton and P. Sutcliffe, Topological Solitons (Cambridge University Press, Cambridge, England, 2007).

[3] A. Vilenkin and E. P. S Shellard, Cosmic Strings and Other Cosmological Defects (Cambridge University Press, Cambridge, England, 1994).

[4] A. A. Abrikosov, J. Phys. Chem. Solids 2, 199 (1957).

[5] N. Nielsen and P. Olesen, Nucl. Phys. B61, 45 (1973).

[6] E. Ayon-Beato, Phys. Rev. D 62, 104004 (2000).

[7] A. Achucarro, R. Gregory, and K. Kuijken, Phys. Rev. D 52, 5729 (1995).

[8] A. M. Ghezelbash and R. B. Mann, Phys. Rev. D 65, 124022 (2002).

[9] M. H. Dehghani and T. Jalali, Phys. Rev. D 66, 124014 (2002).

[10] S. S. Gubser, Phys. Rev. D 78, 065034 (2008).

[11] S. A. Hartnoll, C. P. Herzog, and G. T. Horowitz, Phys. Rev. Lett. 101, 031601 (2008).

[12] G. T. Horowitz and M. M. Roberts, Phys. Rev. D 78, 126008 (2008).

[13] D. Arean, P. Basu, and C. Krishnan, J. High Energy Phys. 10 (2010) 006.

[14] P. Breitenlohner and D. Z. Freedman, Phys. Lett. 115B, 197 (1982); 1042 citations counted in INSPIRE as of 01 .

[15] Y. Brihaye and B. Hartmann, J. High Energy Phys. 09 (2010) 002.

[16] Y. Brihaye and B. Hartmann, Phys. Rev. D 83, 126008 (2011).

[17] F. Canfora, Eur. Phys. J. C 78, 929 (2018).

[18] L. Aviles, F. Canfora, N. Dimakis, and D. Hidalgo, Phys. Rev. D 96, 125005 (2017).

[19] F. Canfora, M. Lagos, S. H. Oh, J. Oliva, and A. Vera, Phys. Rev. D 98, 085003 (2018).

[20] F. Canfora, N. Dimakis, and A. Paliathanasis, Eur. Phys. J. C 79, 139 (2019).

[21] F. Canfora, S. H. Oh, and A. Vera, Eur. Phys. J. C 79, 485 (2019).

[22] F. Canfora, M. Lagos, and A. Vera, Eur. Phys. J. C 80, 697 (2020).

[23] F. Canfora, A. Giacomini, M. Lagos, S. H. Oh, and A. Vera, Eur. Phys. J. C 81, 55 (2021).

[24] E. Witten, Nucl. Phys. B249, 557 (1985).

[25] A. Hanany and D. Tong, J. High Energy Phys. 07 (2003) 037; 04 (2004) 066(E).
[26] R. Auzzi, S. Bolognesi, J. Evslin, K. Konishi, and A. Yung, Nucl. Phys. B673, 187 (2003).

[27] M. Shifman and A. Yung, Phys. Rev. D 70, 045004 (2004).

[28] A. Gorsky, M. Shifman, and A. Yung, Phys. Rev. D 71, 045010 (2005).

[29] M. Eto, M. Nitta, and N. Yamamoto, Phys. Rev. Lett. 104, 161601 (2010).

[30] A. Gorsky, M. Shifman, and A. Yung, Phys. Rev. D 83, 085027 (2011).

[31] R. L. Davis and E. P. S. Shellard, Nucl. Phys. B323, 209 (1989).

[32] R. H. Brandenberger, B. Carter, A. C. Davis, and M. Trodden, Phys. Rev. D 54, 6059 (1996).

[33] A. C. Davis and W. B. Perkins, Phys. Lett. B 393, 46 (1997).

[34] L. Masperi and M. Orsaria, Int. J. Mod. Phys. A 14, 3581 (1999).

[35] L. Masperi and G. A. Silva, Astropart. Phys. 8, 173 (1998).

[36] E. Radu and M. S. Volkov, Phys. Rep. 468, 101 (2008).

[37] Y. Lemperiere and E. P. S. Shellard, Phys. Rev. Lett. 91, 141601 (2003).

[38] Y. Lemperiere and E. P. S. Shellard, Nucl. Phys. B649, 511 (2003).

[39] T. Vachaspati, Phys. Rev. Lett. 101, 141301 (2008).

[40] J. Ye, K. Wang, and Y.-F. Cai, Eur. Phys. J. C 77, 720 (2017).

[41] A. Gruzinov and A. Vilenkin, J. Cosmol. Astropart. Phys. 01 (2017) 029.

[42] I. Yu. Rybak, A. Avgoustidis, and C. J. A. P. Martins, Phys. Rev. D 96, 103535 (2017); 100, 049901(E) (2019).

[43] B. Hartmann, F. Michel, and P. Peter, Phys. Rev. D 96, 123531 (2017).

[44] Y.-W. Yu, K.-S. Cheng, G. Shiu, and H. Tye, J. Cosmol. Astropart. Phys. 11 (2014) 040.

[45] K. Miyamoto and K. Nakayama, J. Cosmol. Astropart. Phys. 07 (2013) 012.

[46] B. Hartmann and F. Michel, Phys. Rev. D 86, 105026 (2012).

[47] Y.-F. Cai, E. Sabancilar, D. A. Steer, and T. Vachaspati, Phys. Rev. D 86, 043521 (2012).

[48] E. Trojan and G. V. Vlasov, Phys. Rev. D 85, 107303 (2012).

[49] E. Ayon-Beato and M. Hassaine, Phys. Rev. D 75, 064025 (2007).

[50] D. E. Berenstein, J. M. Maldacena, and H. S. Nastase, J. High Energy Phys. 04 (2002) 013.

[51] A. Cisterna, M. Hassaine, J. Oliva, and M. Rinaldi, Phys. Rev. D 94, 104039 (2016).

[52] W. S. l'Yi, arXiv:hep-th/9808051.

[53] D. G. Crowdy, Int. J. Eng. Sci. 35, 141 (1997). 
[54] J. Liouville, J. Math. Pure Appl. 1re Ser. 18, 71 (1853).

[55] A. D. Polyanin and V. E. Nasaikinskii, Handbook of Linear Partial Differential Equations for Engineers and Scientists, 2nd ed. (CRC Press, Taylor \& Francis Group, London, 2015).
[56] S. Flügge, in Practical Quantum Mechanics (SpringerVerlag, Berlin, 1971), Vol. 1, p. 94.

[57] F. Cooper, A. Khare, and U. Sukhatme, Phys. Rep. 251, 267 (1995).

[58] M. Ortaggio, Classical Quantum Gravity 35, 127001 (2018). 